\title{
Endoscopic submucosal dissection for a recurrent rectal tumor extending to the dentate line
}

\author{
Viale $\mathrm{E}^{1}$, Maselli $\mathrm{R}^{1,2}$, Notaristefano $\mathrm{C}^{1}$ and Testoni $\mathrm{PA}^{1-3}$ \\ ${ }^{1}$ Gastroenterological and Endoscopic Unit, Division of Experimental Oncology. IRCCS San Raffaele Scientific Institute, Milan, Italy \\ ${ }^{2}$ Surgical Sciences Department, Sapienza University of Rome, Rome, Italy \\ ${ }^{3}$ Gastroenterological and Endoscopic Unit, Division of Experimental Oncology, Vita-salute San Raffaele University, Milan, Italy
}

Endoscopic submucosal dissection (ESD) for rectal tumors extending to the dentate line (RTDL) is considered very challenging even for expert operators, mainly due to anatomical reasons. Fortunately, these lesions are rare, but their treatment remains difficult with a long procedure time and high complication rate and a modified ESD technique for RTDLs has been recently proposed [1].

For these reasons, it is not surprising that most of the studies on RTDL are coming from Japanese centers, where the ESD have been established more than 10 years ago [2].

Even though, also in a European setting it is possible to approach such difficult lesions $[3,4]$. We successfully treated an RTDL previously endoscopically treated (three times) in different centers.

The patient, a 70 years old man, was referred to our center for the treatment of a recurrent granular, mix type LST of $40 \times 25 \mathrm{~mm}$ in length, extending to the dentate line (Figure 1). In particular the

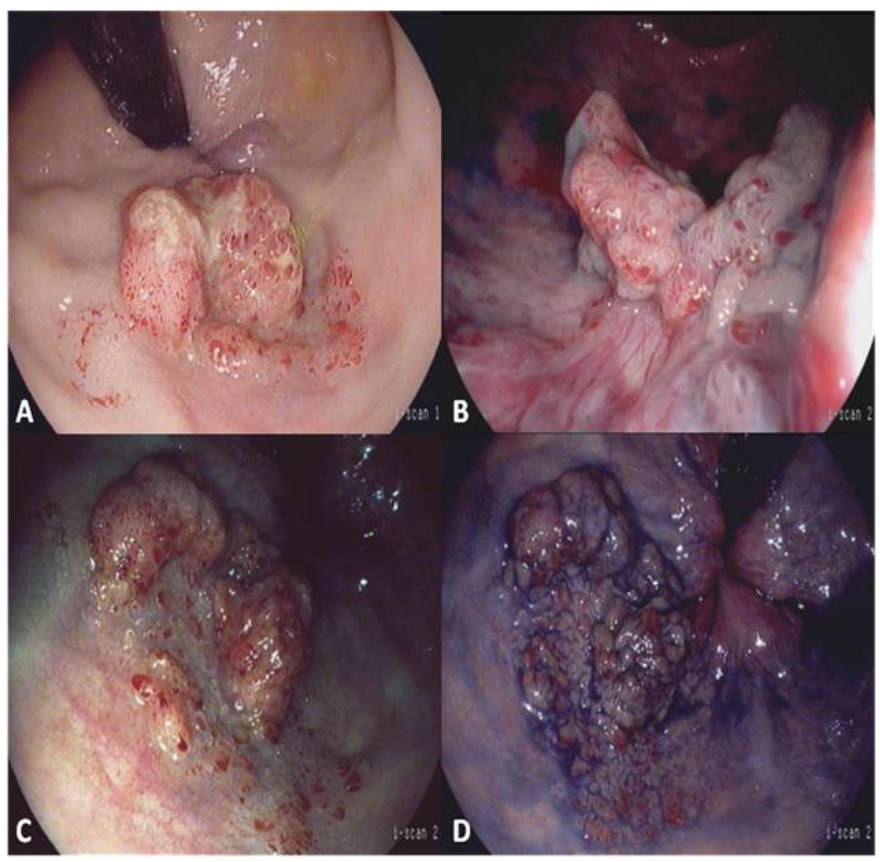

Figure 1. Endoscopic images of a recurrent RTDL. A, B: white light imaged in retroflexion view (A) and front vision (B). C: I-scan 2 retroflexion view showing the RTDL with adenomatous islands on fibrosis. D: same images in B with indigo carmine $0.4 \%$ chromoendoscopy. oral side of the lesion showed adenomatous focal islands on a fibrotic tissue. Considering the closeness of the rectal venous plexus, the fibrovascular submucosa at the anal canal, and the submucosal fibrosis at the proximal edge of the lesion due to the previous treatments, the ESD was very challenging. A gastroscope with waterjet system, HD, I-scan, contrast and tone enhancement (EPK-i, Pentax Medical, Japan), a standard electrosurgical generator (VIO300, ERBE, Germany), two knives (Dual knife and Hook Knife, Olympus, Japan), were used. We

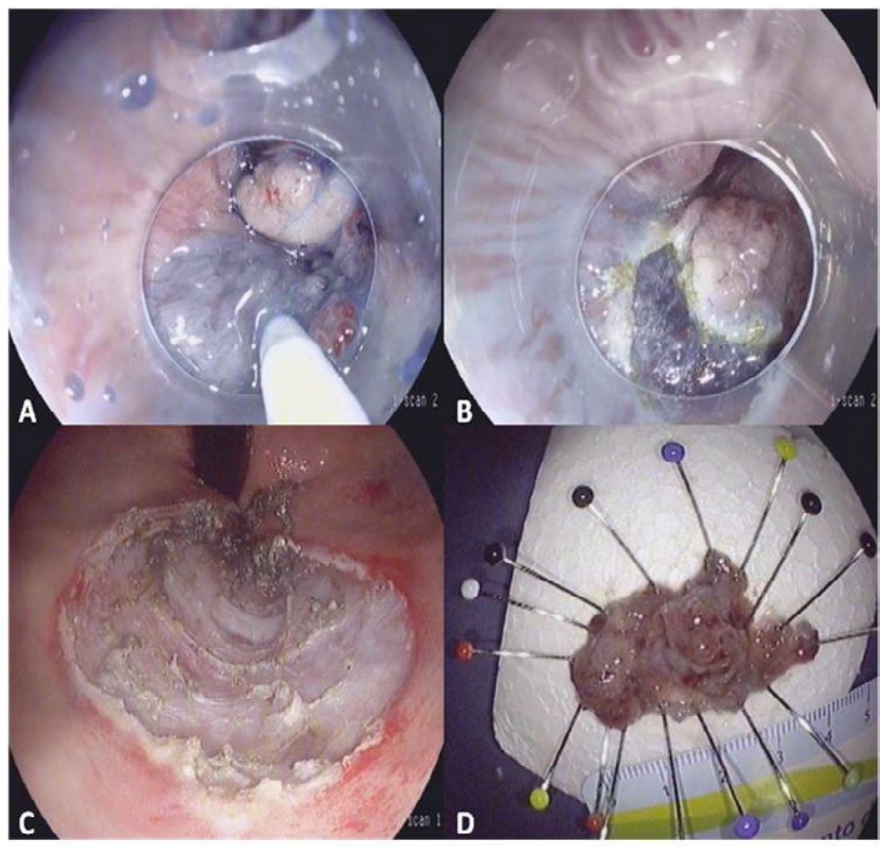

Figure 2. Endoscopic images of the ESD procedure. A: Glicerol + indigo carmine submucosal injection and mucosal incision (B) at the dentate line side. C: Completed ESD, retroflex image. D: fixed ESD specimen with length counter.

Correspondence to: Dr. Roberta Maselli, Gastroenterological and Endoscopic Unit, Division of Experimental Oncology, IRCCS San Raffaele Scientific Institute, Milan, Italy, Tel: +39 3338007368; E-mail: Roberta.maselli.md@gmail.com

Key words: endoscopic submucosal dissection, ESD, rectal tumor

Received: July 17, 2015; Accepted: August 15, 2015; Published: August 18, 2015 
did not inject lidocaine in the submucosa, because the procedure was performed with deep sedation, administered by an anesthesiologist, and no pain was felt by the patient during the treatment.

An en bloc resection, R0, uncomplicated procedure was performed (Figure 2).

In conclusion, we directly validated the feasibility and the safety of ESD for RTDLs, even for those lesions that recur after not radical treatments.

\section{References}

1. Imai K, Hotta K, Yamaguchi Y, Shinohara T, Ooka S, et al. (2015) Safety and efficacy of endoscopic submucosal dissection of rectal tumors extending to the dentate line. Endoscopy 47: 529-532. [Crossref]

2. Watanabe T, Sawada T, Saito Y, Sunouchi K, Masaki T, et al. (1995) Endoscopic treatment of colorectal cancer. GanTo Kagaku Ryoho 22: 202-208. [Crossref]

3. Spychalski M, Dziki A (2014) Safe and efficient colorectal endoscopic submucosal dissection in European settings: Is successful implementation of the procedure possible? Dig Endosc. [Crossref]

4. Neuhaus H (2014) ESD around the world: Europe. Gastrointest Endosc Clin N Am 24: 295-311. [Crossref]

Copyright: $(02015$ Viale E. This is an open-access article distributed under the terms of the Creative Commons Attribution License, which permits unrestricted use, distribution, and reproduction in any medium, provided the original author and source are credited. 